\title{
Relación entre características del hábitat y estructura del ensamble de insectos en humedales palustres urbanos del centro-sur de Chile
}

\author{
Relationship between habitat characteristics and insect assemblage structure in urban \\ freshwater marshes from central-south Chile
}

ROMINA VILLAGRÁN-MELLA ${ }^{1 *}$, MAURICIO AGUAYO², LUIS E. PARRA \& ANGÉLICA GONZÁLEZ ${ }^{3}$

\author{
${ }^{1}$ Departamento de Zoología, Facultad de Ciencias Naturales y Oceanográficas, \\ Universidad de Concepción; luparra@udec.cl \\ ${ }^{2}$ Centro EULA, Universidad de Concepción; maaguayo@udec.cl \\ ${ }^{3}$ Centro de Estudios Avanzados en Ecología y Biodiversidad (CASEB) \& Departamento de Ecología, \\ Facultad de Ciencias Biológicas, Pontificia Universidad Católica de Chile; agonzag@bio.puc.cl \\ * e-mail para correspondencia: rvillagr@bio.puc.cl
}

\begin{abstract}
RESUMEN
Los humedales son ecosistemas altamente productivos que se caracterizan por cumplir múltiples funciones, manteniendo además una alta diversidad biológica. No obstante, casi la mitad de los humedales en el mundo ha desaparecido en el último siglo debido al proceso de desarrollo urbano. En Chile, la diversidad bioclimática genera una gran variedad de ambientes acuáticos. Estos ecosistemas también han sido expuestos a fuertes presiones antrópicas, debido al proceso de urbanización. En la intercomuna Concepción-TalcahuanoSan Pedro (Región del Biobío) más del $23 \%$ (1.734 ha) del área ocupada por humedales se ha perdido en las últimas tres décadas. Evaluamos la relación entre características del hábitat (morfométricas, limnológicas y vegetacionales) y la estructura del ensamble de insectos en siete humedales palustres de la intercomuna, con el fin de determinar los efectos de la urbanización sobre los patrones de diversidad en estos ecosistemas. Los análisis mostraron que la abundancia relativa y la diversidad de especies se relacionan positivamente con la naturalidad de la matriz y el contenido de oxígeno en el agua, esta última característica fue el predictor más importante para la estructura del ensamble de insectos. De las 24 morfoespecies de insectos analizadas, la abundancia de siete especies fue relacionada significativamente con las características del hábitat consideradas. La naturalidad de la matriz, el área del humedal, la heterogeneidad vegetacional y la concentración de oxígeno disuelto se relacionaron positivamente con la abundancia de estas especies, mientras que la conductividad y/o turbidez del agua mostró un efecto negativo sobre ella. La disminución de la diversidad de insectos determinada por características del hábitat asociadas con la pérdida, fragmentación, homogeneización, eutroficación y/o contaminación de los humedales indica que los insectos podrían ser utilizados como indicadores de los efectos de la urbanización sobre el funcionamiento de estos ecosistemas. Sin embargo, dada la falta de información biológica y taxonómica en especies de insectos asociados a humedales palustres, solo características del hábitat con efectos significativos a un nivel taxonómico alto podrían ser consideradas para establecer recomendaciones iniciales de planes de manejo.
\end{abstract}

Palabras clave: conservación, humedales, urbanización, fragmentación del hábitat, calidad del agua, biodiversidad, insectos, Chile.

\begin{abstract}
Wetlands are one of the most productive ecosystems which provide a number of ecosystem functions, maintaining also a high biodiversity. Nevertheless, almost half of the wetlands in the world have disappeared in the last century due to urban development process. Along the Chilean landscape a great variety of aquatic habitats exist. Due to urban expansion those ecosystems have been exposed to strong anthropogenic pressures. In the intercomunal area Concepción-Talcahuano- San Pedro (Biobío Region), more than $23 \%$ (1,734 ha) of the wetland areas have been lost in the last three decades. We evaluated the relationship between habitat characteristics (morphometric, limnology and vegetation) and the insect assemblage's structure in seven freshwater marshes in this intercomunal area. Our aim was to assess the influence of urbanization on the diversity patterns of these ecosystems. Insect abundance and species diversity were positively correlated to matrix pristinness and oxygen concentration of the water, this last feature was the best predictor for the structure of the insect assemblage. Of the 24 insect morphospecies included in the analysis, the abundance of only seven species was significantly related to the quantified habitat characteristics. Matrix pristinness,
\end{abstract}


wetland area, vegetation heterogeneity and water oxygen concentration were positively related to species abundance, however, conductivity and water density showed a negative effect on the abundance. The insect species diversity decrease determined by the habitat characteristics associated to habitat loss, habitat fragmentation, habitat homogeneity, and wetland contamination, show that insects could be used as indicators of the urban effects on the functioning of these ecosystem. However, due to the missing biological and taxonomical information on the insect species related to freshwater marshes, only habitat characteristics with a significant effect on a high taxonomical level could be considered to establish initial recommendations for management plans.

Key words: conservation, wetlands, urbanization, habitat fragmentation, water quality, biodiversity, insects, Chile.

\section{INTRODUCCIÓN}

La urbanización es un proceso continuo que ha producido alteraciones en la estructura y funcionamiento de los ecosistemas (McDonnell \& Pickett 1990, Green \& Baker 2003, Takami et al. 2004). Este proceso está asociado a incrementos en la exposición a contaminantes, eutroficación, alteraciones en la hidrología y en la estructura del paisaje (Ehrenfeld 2004, Kentula et al. 2004, Rubbo \& Kiesecker 2005). El impacto del desarrollo urbano sobre la biota provoca una disminución de la biodiversidad y cambios en la composición de especies (McDonnell \& Pickett 1990, Gleason et al. 2002, Hunter 2002). De esta manera, los efectos de la urbanización sobre la diversidad biológica están fuertemente unidos a los atributos de los ecosistemas en distintas escalas espaciales (Rubbo \& Kiesecker 2005). Estos efectos son particularmente fuertes en los ecosistemas de humedal, debido a que la escasez de áreas para desarrollo urbano ha provocado la destrucción de pequeños humedales alrededor del mundo (Holland et al. 1995, Gallego et al. 1999, Semlitsch \& Bodie 1998).

Muchas de las especies que dependen de los ecosistemas de humedal están dentro de los grupos más amenazados de extinción (Ricciardi et al. 1998). En este contexto, el uso de indicadores biológicos en el monitoreo de cambios en las condiciones del hábitat, constituye una herramienta útil en la prevención de pérdida de especies (Summerville et al. 2004). Dentro de los invertebrados, los insectos han sido considerados excelentes indicadores biológicos, dado que poseen requerimientos ecológicos específicos y ciclos de vida cortos (Brown 1997, Kitahara \& Sei 2001, Figueroa et al. 2003, Maes et al. 2005).
En ecosistemas terrestres, afectados por la pérdida, alteración o fragmentación del hábitat, se ha encontrado una relación positiva entre la diversidad de especies de insectos y una serie de atributos de los ecosistemas, tales como el área e irregularidad en la forma de los parches de hábitat, la naturalidad de la matriz y la heterogeneidad vegetacional (Grez 1992, Dennis et al. 1998, Steffan-Dewenter \& Tscharntke 2000, Barbosa \& Marquet 2002). En ecosistemas de humedal, se ha sugerido una relación positiva entre la riqueza de especies de insectos y la naturalidad de la matriz (Anderson \& Vondracek 1999, Lundkvist et al. 2001), la heterogeneidad vegetacional (Cronin et al. 1998, Voelz \& Mcarthur 2000, Brose 2003), la profundidad de los humedales (Leslie et al. 1997, Brooks $2000,2002)$ y la calidad del agua (Spieles \& Mitsch 2000, Gleason et al. 2003, Figueroa et al. 2003). Sin embargo, pocos estudios han considerado el conjunto de estas características del hábitat y su relación con la estructura del ensamble de insectos, con el propósito de evaluar los efectos de la urbanización sobre humedales (Pauchard et al. 2005). Pocos investigadores han identificado además la respuesta a nivel específico frente a cambios en los atributos del hábitat (Gibson et al. 1992, Wettstein \& Schmid 1999).

La diversidad bioclimática de Chile genera una gran variedad de ambientes acuáticos. Los lagos y lagunas de la zona central se caracterizan por ser altamente productivos, de escasa profundidad, con vegetación ribereña alta casi en todo su perímetro y abundante vegetación sumergida (Riveros et al. 1981). Debido a la expansión urbana, los humedales han sido expuestos a fuertes presiones antrópicas, siendo reducidos en extensión o transformados para actividades de construcción (Parra et al. 1989). Existe un creciente interés 
en el uso de humedales para el desarrollo de proyectos de expansión urbana e industrial, a pesar de la falta de información sobre el impacto ambiental en estos ecosistemas y su biota (Parra et al. 1989, González \& Victoriano 2005, Pauchard et al. 2005).

En el centro-sur de Chile, la intercomuna Concepción- Talcahuano-San Pedro (Región del Biobío) constituye una de las áreas con mayor número de humedales insertos en el radio urbano (Fig. 1) (González \& Victoriano 2005). En esta zona, más del $23 \%$ (1.734 ha) del área ocupada por humedales se ha perdido en las últimas tres décadas producto de la expansión urbana, resultando en humedales con distintos grados de fragmentación, pérdida y alteración del hábitat (Pauchard et al. 2005).
En este trabajo se propone que la diversidad de especies de insectos (riqueza de especies y abundancia relativa) decrece con la disminución del área, regularidad en la forma del humedal, naturalidad de la matriz, heterogeneidad vegetacional, profundidad y calidad del agua de los humedales. Por otra parte, se espera que las especies de insectos respondan diferencialmente a los distintos atributos del hábitat, mediante disminuciones en su abundancia relativa.

El objetivo del presente estudio fue relacionar la estructura del ensamble de insectos y las características del hábitat en humedales palustres de la intercomuna Concepción-Talcahuano-San Pedro, con el fin de determinar los efectos de la urbanización sobre los patrones de diversidad de estos sistemas ecológicos.

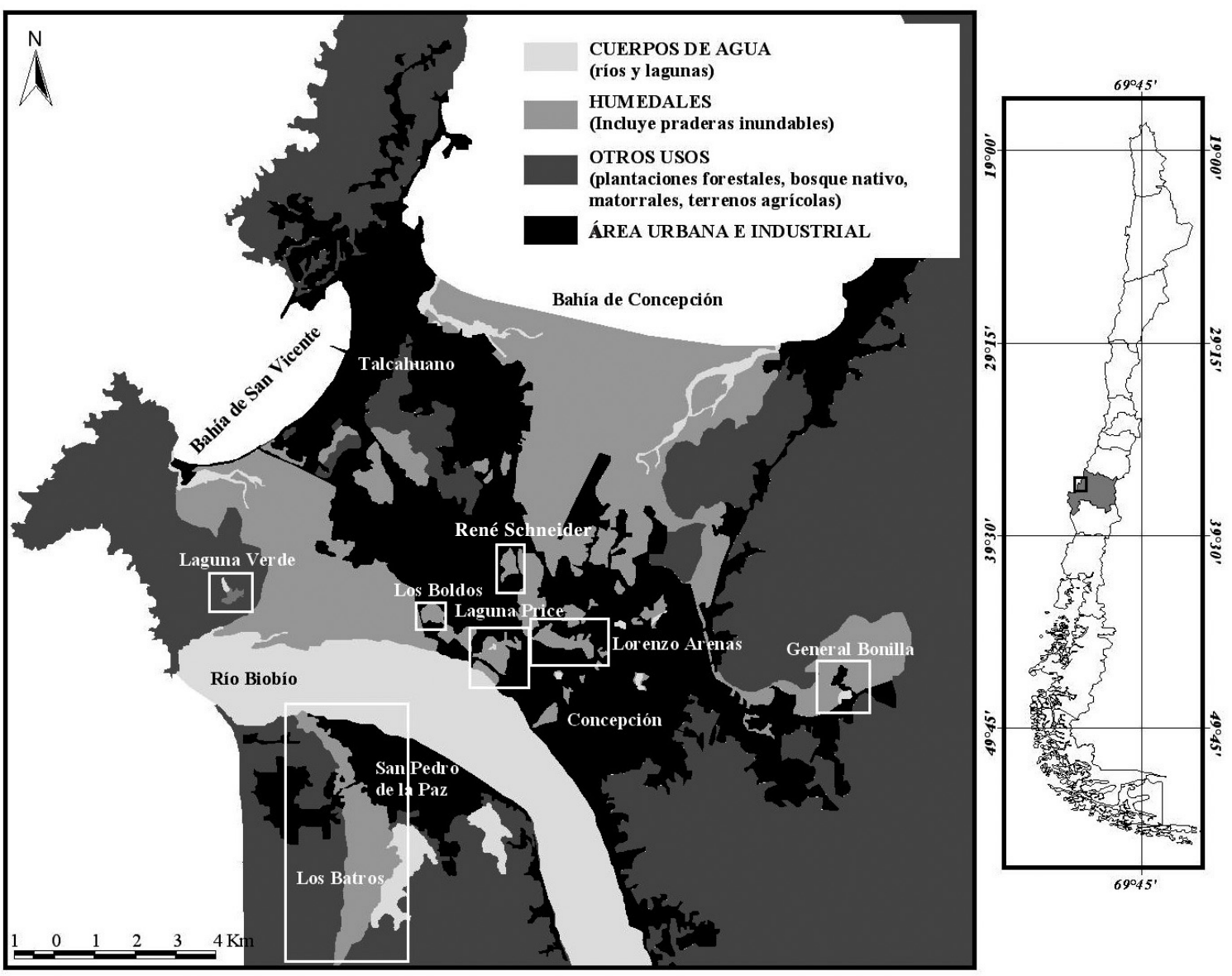

Fig. 1: Área de estudio: ubicación de los humedales en la intercomuna Concepción-Talcahuano-San Pedro (Región del Biobío, Chile).

Study area: wetlands location in the intercomunal area Concepción-Talcahuano-San Pedro (Biobío Region, Chile). 


\section{MATERIALES Y MÉTODOS}

\section{Área de estudio}

El estudio se llevó a cabo en las comunas de Concepción, Talcahuano y San Pedro, localizadas en la zona costera de la Región del Biobío, Chile ( $\left.36^{\circ} 46^{\prime} \mathrm{S}, 73^{\circ} 02^{\prime} \mathrm{O}\right)$. El clima es de tipo templado-cálido, con medias anuales de $12,4{ }^{\circ} \mathrm{C}$ de temperatura, $1.235 \mathrm{~mm}$ de precipitaciones y $81 \%$ de humedad relativa (Riffo \& Villarroel 2000). Se estudiaron siete humedales palustres: Laguna Verde, Laguna Price, Los Boldos, René Schneider, Lorenzo Arenas, General Bonilla y Los Batros (Fig. 1). El humedal Los Batros se ubica al sur del río Biobío y se originó a partir del represamiento de una subcuenca por el depósito de arenas del mismo río, mientras el resto de los humedales se encuentran al norte del río y se originaron a partir de la depresión de su antiguo cauce (González \& Victoriano 2005). Las especies vegetales dominantes en este tipo de humedales son: Juncus procerus, Scirpus californicus, Cyperus eragrostis, Juncus imbricatus y Typha domingensis (Polymerys 1995, Riffo \& Villarroel 2000).

De estos ecosistemas, Laguna Verde constituye el único humedal alejado del núcleo urbano (Fig. 1). Ubicado dentro de la Península de Hualpén, se encuentra inserto en una matriz de bosque nativo donde entran en contacto especies de bosque esclerófilo con especies del bosque costero higrófilo. El bosque nativo, dominado por Cryptocarya alba (peumo), Aextoxicon punctatum (olivillo), Lithrea caustica (litre) y Peumus boldus (boldo), carece de representantes del género Nothofagus e incluye como especies menos abundantes a Drimys winteri (canelo), Eucryphia cordifolia (ulmo) y Laurelia sempervirens (laurel) (Polymerys 1995).

\section{Características del hábitat}

Cada humedal fue estudiado entre octubre del año 2003 y enero del 2004. La caracterización de los humedales fue realizada mediante la cuantificación de 16 características morfométricas, vegetacionales y físicoquímicas (Tabla 1). El área, forma y naturalidad de la matriz fueron estimadas a partir de fotografías aéreas escala 1:10.000 tomadas el año 2003. Estas fueron georeferenciadas y analizadas por medio del programa ArcView 3.3. Para la forma del humedal, se calcularon los índices de diversidad de Patton $\left(\mathrm{R}=\mathrm{p} / 2(\pi \mathrm{A} 1)^{1 / 2}\right)$ y de compactación de Unwin $(\mathrm{K}=1 / \mathrm{R})$ (Rau \& Gantz 2001), donde p y A corresponden al perímetro y al área del parche de hábitat, respectivamente. El valor de $\mathrm{R}$ varía entre $1 \mathrm{e}$ infinito, es invariante de escala y puede clasificarse en los siguientes rangos de clase: desde fragmentos redondos $(\mathrm{R}<1,25)$ a irregulares $(\mathrm{R}>2,00)$. $\mathrm{K}$, toma el valor de 0 para parches de hábitat con mayor exposición periférica a la matriz y 1 para parches compactos menos expuestos (Rau \& Gantz 2001).

La naturalidad de la matriz fue estimada a partir del porcentaje de "área natural” o área no urbanizada, considerando una zona buffer de 1 $\mathrm{km}$ de radio alrededor del borde de cada humedal. Se clasificaron como "áreas naturales" a praderas inundables, lagunas y parches de vegetación nativa y, como "áreas no naturales" a carreteras, edificios y plantaciones forestales (Simonetti 1999, Marzluff \& Ewing 2001).

La heterogeneidad de hábitat fue calculada a partir del Índice de Diversidad de ShannonWiener usando el porcentaje de cobertura de las especies de plantas acuáticas, utilizando cinco parcelas de $1 \mathrm{~m}^{2}$ instaladas en las mismas zonas de muestreo de insectos. La profundidad de los humedales fue medida en 30 puntos de muestreo mediante el uso de limnímetros. La calidad del agua fue estimada a través de parámetros físicos y químicos del agua (concentración de oxígeno disuelto, temperatura, turbidez, $\mathrm{pH}$, conductividad, DQO y concentración de nutrientes) en los mismos puntos de muestreo. El grado de turbidez del agua se estimó a través de la profundidad de alcance de la luz según visión disco Secchi, expresada en $\mathrm{cm}$ y multiplicada por la constante empírica de ajuste $(\mathrm{C}=1,7)$ (Margalef 1983). Los valores de DQO, Nitrato y Fosfato fueron obtenidos en laboratorio, para lo cual se tomaron muestras de agua en recipientes de 1 L mantenidos a baja temperatura en cinco puntos por humedal (Conzonno 1995). 


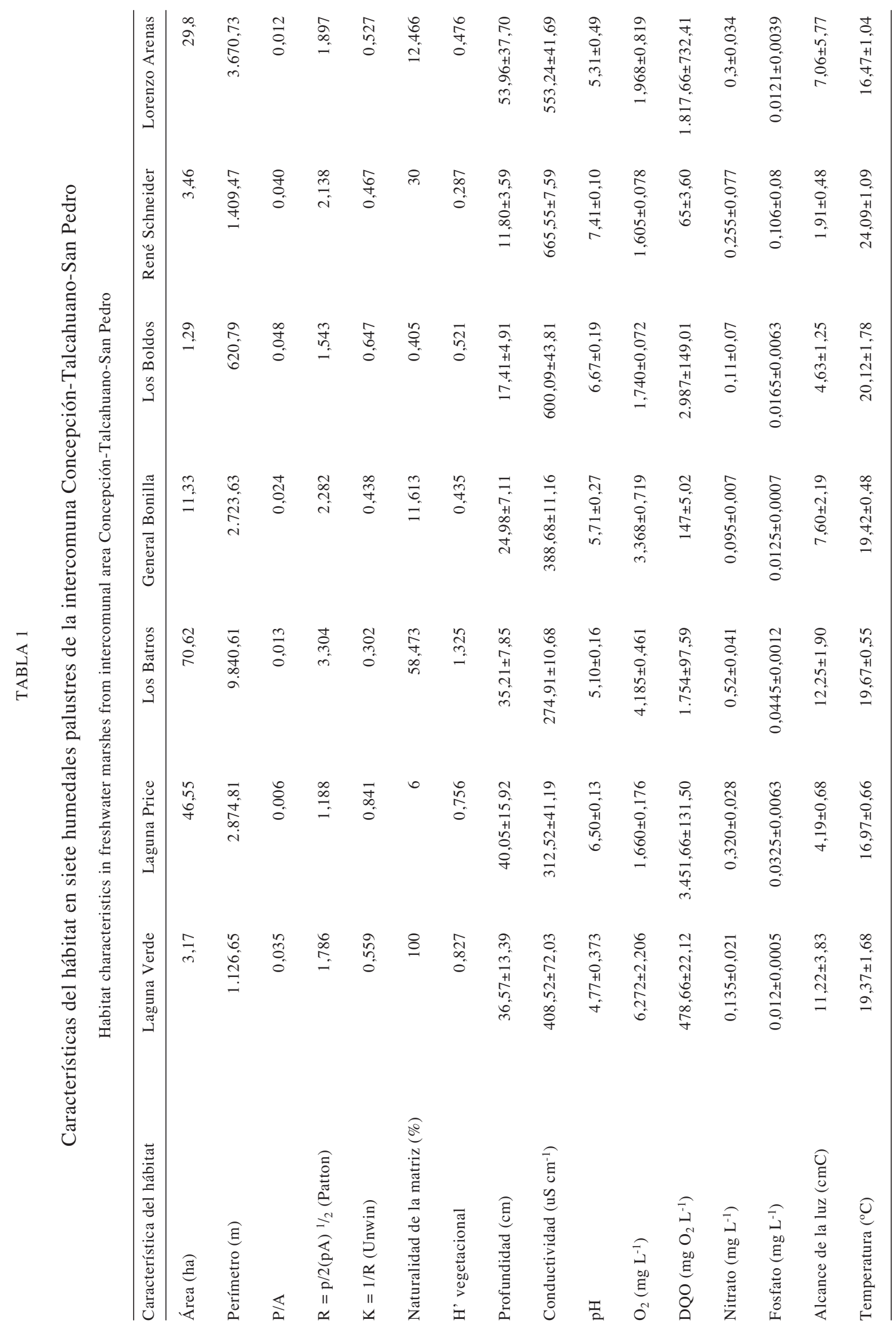




\section{Ensambles de insectos}

Los insectos fueron colectados entre octubre del año 2003 y enero del 2004, en 30 puntos de muestreo distribuidos al azar a partir de 3 metros del borde de cada humedal. La captura se realizó entre la vegetación emergente por medio de redes entomológicas acuáticas para sustratos anegados (BioQuip ${ }^{\circledR}$ products). Las muestras fueron llevadas posteriormente al laboratorio en frascos plásticos, y los ejemplares de insectos adultos e inmaduros fueron separados por morfoespecie y determinados hasta la categoría taxonómica más baja posible. Se cuantificó la abundancia de cada especie y la diversidad (riqueza y abundancia relativa) del ensamble de insectos para cada muestra por humedal. La diversidad de especies fue estimada a partir del índice de Simpsom (D') (Krebs 1999), utilizando las abundancias de cada especie (Biodiversity Profesional 2.0).

\section{Análisis de información}

Se realizó estadística descriptiva básica (promedio, desviación estándar y transformación de datos) para la totalidad de los datos. Para analizar la riqueza de especies se trazaron curvas de acumulación y rarefacción basadas en el número de muestras. Las curvas fueron obtenidas utilizando el programa EstimateS 7.5, considerando 50 iteraciones y usando el método de Coleman (Gotelli \& Colwell 2001). Se comparó la diversidad (riqueza de especies y abundancias relativas) entre humedales mediante análisis de varianza de una vía, seguido por una prueba de comparaciones múltiple de Tukey (programa STATISTICA 6.0).

A través de análisis de regresiones lineales simples se estimó la relación entre variables predictoras (área, perímetro, p/A, R, K, \% naturalidad de la matriz, heterogeneidad vegetacional, profundidad, conductividad, $\mathrm{pH}$, temperatura, oxígeno disuelto, DQO, nitrato, fosfato, profundidad de penetración de la luz) y variables respuestas. Además, se construyó una matriz de correlación de Pearson para cuantificar la correlación entre las variables predictoras. Posteriormente se realizó un análisis de regresión múltiple paso a paso ("stepwise multiple regression") para determinar las características del hábitat que mejor predicen la estructura del ensamble de insectos (programa SPSS 10.0). Las gráficas fueron elaboradas en el programa SigmaPlot 8.0.

\section{RESULTADOS}

Los humedales presentaron un gradiente a nivel de área, irregularidad en la forma de los fragmentos, naturalidad de la matriz y heterogeneidad vegetacional (Tabla 1). Estos mostraron además diferencias significativas en profundidad $\left(\mathrm{F}_{1,6}=140,3 ; \mathrm{P}<0,01\right)$ y en todos los parámetros físicoquímicos del agua; oxígeno $\left(\mathrm{F}_{1,6}=143,8 ; \mathrm{P}<0,01\right)$, conductividad $\left(\mathrm{F}_{1,6}=627,6 ; \mathrm{P}<0,01\right)$, temperatura $\left(\mathrm{F}_{1,6}=\right.$ 232,8; $\mathrm{P}<0,01)$, profundidad de alcance de la luz $\left(\mathrm{F}_{1,6}=104,8 ; \mathrm{P}<0,01\right), \mathrm{pH}\left(\mathrm{F}_{1,6}=621,2 ; \mathrm{P}\right.$ $<0,01)$, DQO $\left(\mathrm{F}_{1,6}=125,1 ; \mathrm{P}<0,01\right)$, nitrato $\left(\mathrm{F}_{1,6}=20,27 ; \mathrm{P}<0,01\right)$ y fosfato $\left(\mathrm{F}_{1,6}=20,3\right.$; $\mathrm{P}<0,01)$. El análisis de correlación entre las características del hábitat indicó una relación positiva entre: la naturalidad de la matriz y la concentración de oxígeno disuelto $(r=0,82)$, el área del humedal y la heterogeneidad vegetacional $(\mathrm{r}=0,76)$, y la concentración de oxígeno disuelto con la profundidad de penetración de la luz $(\mathrm{r}=0,75)$.

Se recolectó un total de 5.849 individuos distribuidos en 24 morfoespecies, 21 familias y seis órdenes de insectos en el total de muestras y humedales (Tabla 2). La tendencia a la asíntota de las curvas de acumulación de especies y el análisis de las curvas de rarefacción indican que el esfuerzo de muestreo (30 puntos) fue suficiente para representar el número de especies presentes en los humedales (Fig. 2). La riqueza de especies total en los humedales fluctuó entre 14 y 18, la abundancia total entre 208 y 1.940 individuos y la diversidad de especies total (D') entre $D^{\prime}=0,130$ y D' $=0,432$. Tanto la riqueza y diversidad de especies (D') como la abundancia relativa de insectos mostró diferencias significativas entre humedales $\left(\mathrm{F}_{1,6}\right.$ $=13,6 ; \mathrm{P}<0,01 ; \mathrm{F}_{1,6}=10,05 ; \mathrm{P}<0,01$ y $\mathrm{F}_{1,6}=$ 20,4; $\mathrm{P}<0,01$, respectivamente) (Fig. 3). Laguna Verde fue el humedal que presentó los valores más altos de diversidad (Fig. 3). La abundancia relativa por especie solo mostró diferencias significativas entre humedales para Hidroporini gen. sp. $\left(\mathrm{F}_{1,6}=6,10 ; \mathrm{P}<0,01\right)$, Hydrochus sp. $\left(\mathrm{F}_{1,6}=31,2 ; \mathrm{P}<0,01\right)$, Gymnochthebius sp. $\left(\mathrm{F}_{1,6}=55,5 ; \mathrm{P}<0,01\right)$, 
Chrironomiidae gen. sp. $\left(\mathrm{F}_{1,6}=3,7 ; \mathrm{P}<0,01\right)$, Belostoma sp. $\left(\mathrm{F}_{1,6}=3,1 ; \mathrm{P}<0,01\right)$, Ectemnostega $\mathrm{sp} .\left(\mathrm{F}_{1,6}=8,6 ; \mathrm{P}<0,01\right)$ y Verger sp. $\left(\mathrm{F}_{1,6}=4,31 ; \mathrm{P}<0,01\right)$.

\section{TABLA 2}

Composición taxonómica del ensamble de insectos en humedales palustres de la intercomuna Concepción-Talcahuano-San Pedro

Insect assemblage taxonomic composition in freshwater marshes from intercomunal area Concepción-TalcahuanoSan Pedro

\begin{tabular}{|c|c|c|}
\hline Orden & Familia & Especie \\
\hline \multirow[t]{2}{*}{ Collembola } & Sminthuridae & Sminthurides sp. \\
\hline & Isotomidae & Isotoma $\mathrm{sp}$. \\
\hline \multirow[t]{2}{*}{ Odonata } & Aeshnidae & Aeshna sp. \\
\hline & Coenagrionidae & gen. sp. \\
\hline \multirow[t]{5}{*}{ Hemiptera } & Belostomatidae & Belostoma sp \\
\hline & Hydrometridae & Hydrometra sp. \\
\hline & Vellidae & Microvellia sp. \\
\hline & Corixidae & Ectemnostega sp. \\
\hline & Cicadellidae & gen. sp. \\
\hline \multirow[t]{9}{*}{ Coleoptera } & Dytiscidae & gen. sp. 1 \\
\hline & Dytiscidae & Hydroporini gen. sp. \\
\hline & Hydrochidae & Hydrochus sp. \\
\hline & Hydrophilidae & gen. sp. 1 \\
\hline & Hydrophilidae & Helochares sp. \\
\hline & Hydrophilidae & Hydrophilus sp. \\
\hline & Hydraenidae & Gymnochthebius sp. \\
\hline & Scirtidae & Cyphon sp. \\
\hline & Carabidae & Mimodromius sp. \\
\hline \multirow[t]{5}{*}{ Diptera } & Chironomidae & gen. sp. \\
\hline & Culicidae & Culex sp. \\
\hline & Ephydridae & gen. sp. \\
\hline & Dolicopodidae & gen. sp. \\
\hline & Stratyomidae & gen. sp. \\
\hline Trichoptera & Limnephilidae & Verger sp. \\
\hline
\end{tabular}

Contrariamente a lo esperado, la riqueza de especies no se relacionó significativamente con ninguna de las características del hábitat medidas. La abundancia relativa y la diversidad de especies de insectos (D') se relacionaron positivamente con la naturalidad de la matriz y la concentración de oxígeno disuelto (Fig. 4 y 5). La concentración de oxígeno disuelto fue seleccionada como la característica del hábitat que presenta mayor relación con la abundancia y diversidad de especies (D') de insectos (Tabla 3).

Los análisis de regresión, basados en la abundancia relativa por especie (24 especies), indican que solo tres atributos morfométricos: \% de naturalidad de la matriz, el área del humedal y la heterogeneidad vegetacional, junto a tres parámetros físicoquímicos: concentración de oxígeno disuelto, conductividad y profundidad de alcance de la luz, fueron predictores significativos de la abundancia relativa de siete especies de insectos (Tabla 4). La abundancia de 17 especies no fue relacionada significativamente a ninguna de las características del hábitat cuantificadas. La naturalidad de la matriz, concentración de oxígeno disuelto y heterogeneidad vegetacional fueron los mejores predictores de la abundancia relativa a nivel de especies de insectos (Tabla 3 ).

\section{DISCUSIÓN}

\section{Naturalidad de la matriz.}

La disminución en la diversidad de especies de insectos (D') y de la abundancia relativa de especies como Hidroporini gen. sp., Hydrochus sp., Gymnochthebius sp., Chrironomiidae gen. sp. y Verger sp., en relación a un menor \% de naturalidad de la matriz de los humedales, es consistente con lo encontrado por Wettstein y Schmid (1999). Estos autores postulan que la diversidad de insectos es menor en humedales insertos en una matriz no natural, porque esta representa una pérdida de hábitat de humedal potencialmente utilizable por estas especies. Así mismo, Lundkvist et al. (2001) encuentran una disminución similar en la abundancia total del ensamble de coleópteros acuáticos relacionado a la pérdida de naturalidad en la matriz. Gleason et al. (2003) estudiando invertebrados en humedales de pradera, afectados por una alta actividad agrícola, proponen que el aporte de sedimentos desde la matriz provoca la disminución en la abundancia de ciertas especies de insectos acuáticos al sepultar sus bancos de huevos. Por esta razón, los insectos asociados a la columna de agua han sido utilizados como indicadores del tipo de uso del suelo en la matriz circundante a los humedales (Anderson \& Vondracek 1999). En este estudio, la naturalidad de la matriz fue el factor más importante para determinar la abundancia de Hydrochus sp. y Verger sp., y si bien no se puede establecer como causa directa de la disminución de sus abundancias, la relación significativa entre la naturalidad de la matriz y la concentración de oxígeno disuelto indicaría que actividades relacionadas a la urbanización en la matriz podrían estar provocando la eutroficación artificial de los humedales. 

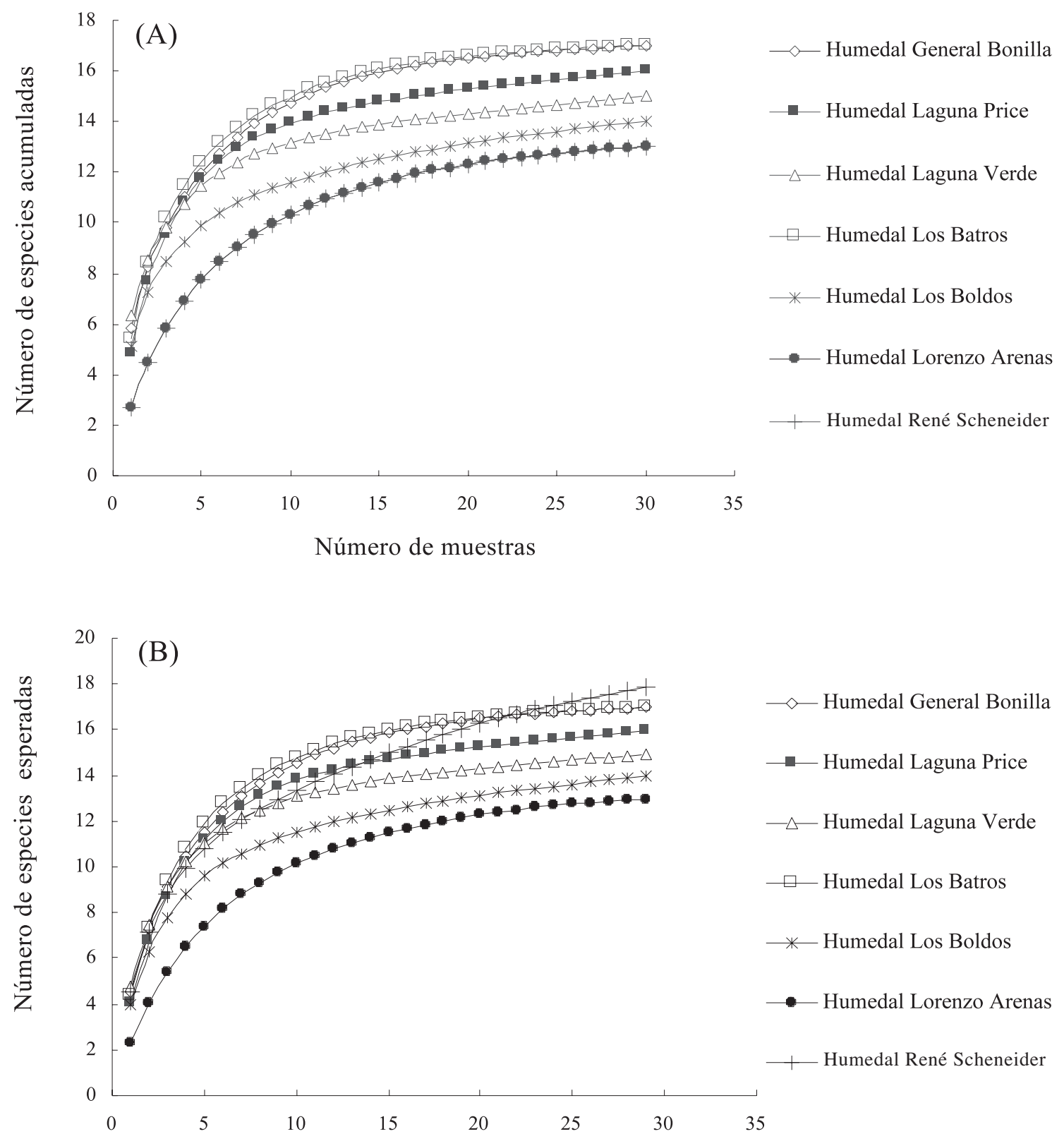

Número de muestras

Fig. 2: (A) Curvas del número acumulado de especies de insectos en 30 muestras por humedal. (B) Curvas de rarefacción de Coleman para el número de especies de insectos en 30 muestras por humedal.

(A) Cumulative number of insect species curves in 30 samples per wetland. (B) Coleman's rarefaction curves for the number of species of insects in 30 samples by wetland. 
(A)

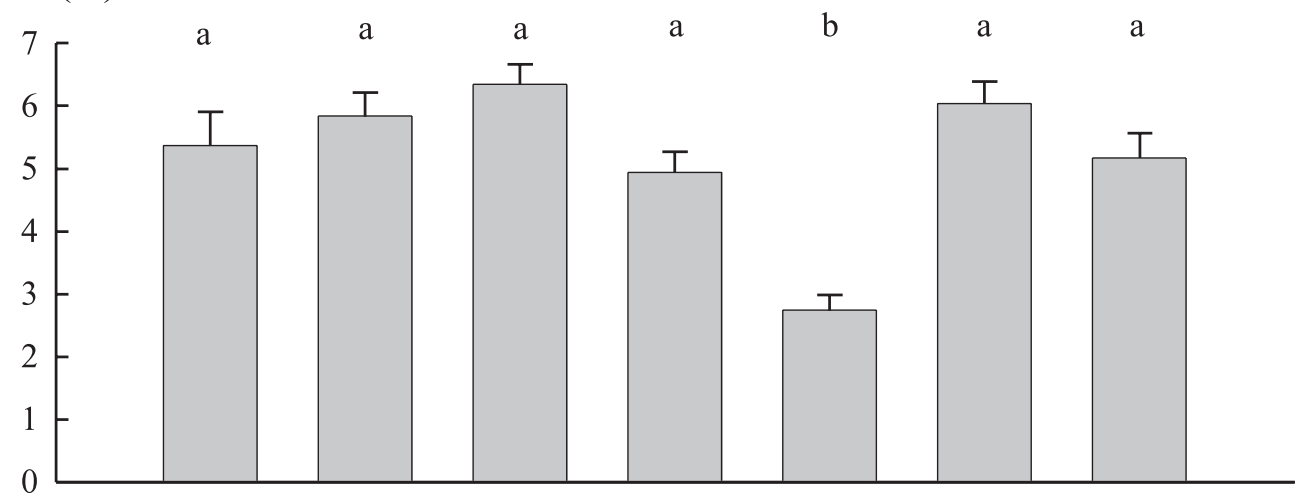

(B)

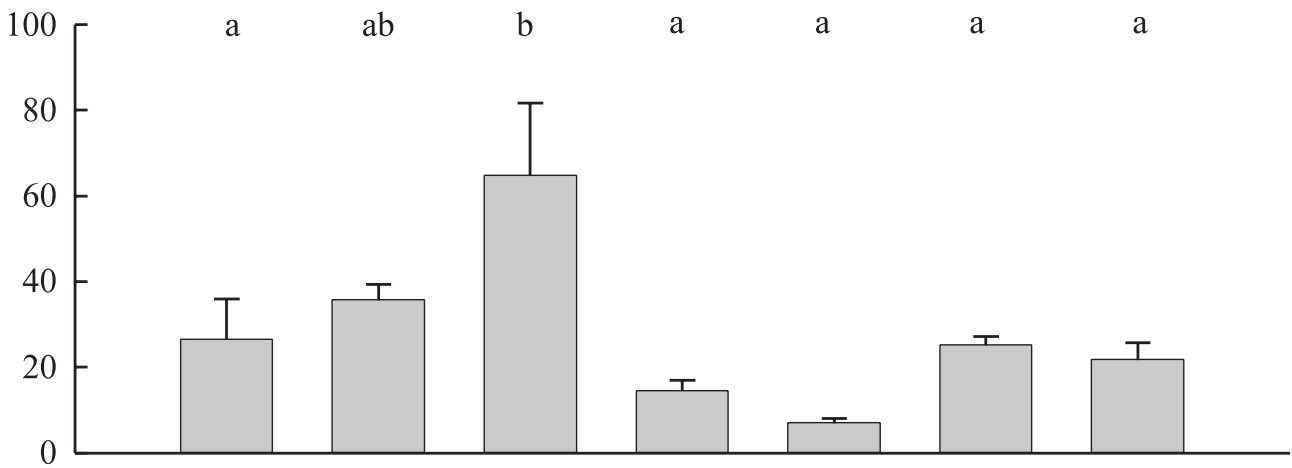

(C)

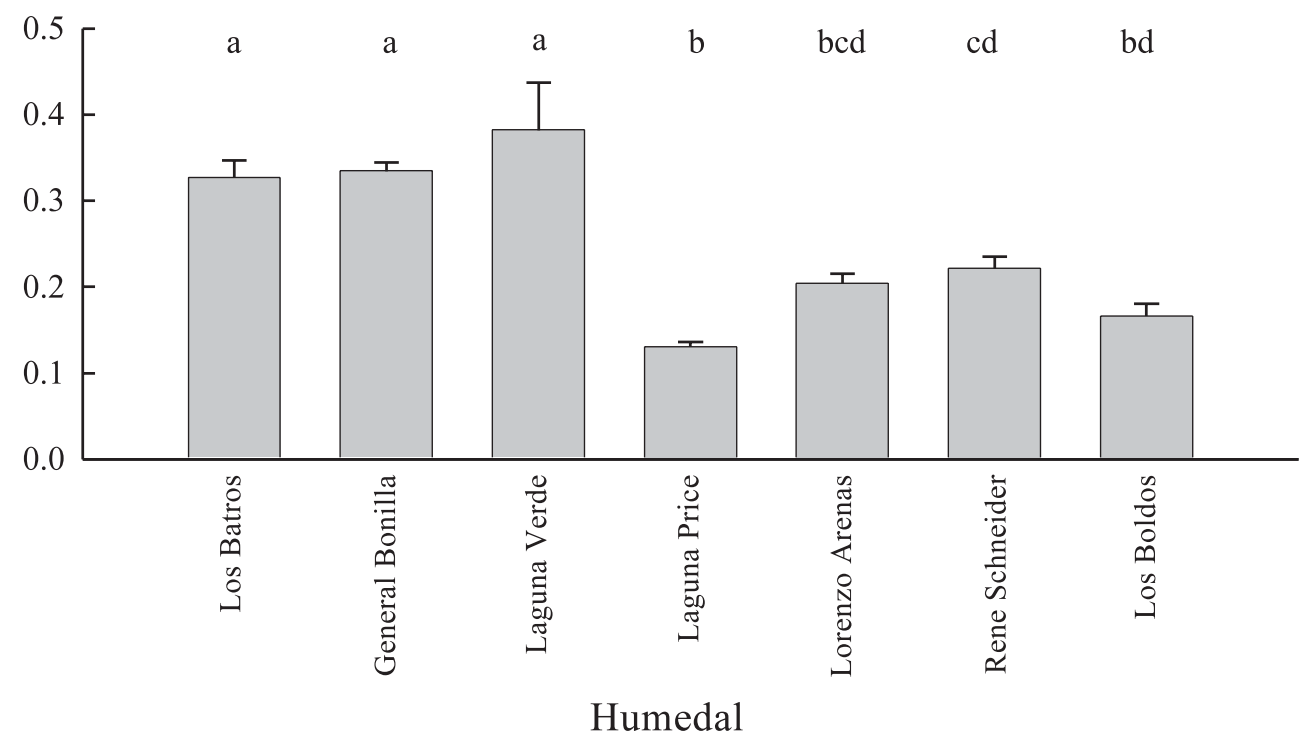

Fig. 3: (A) Riqueza de especies de insectos por humedal (promedio \pm DE). (B) Abundancia de insectos por humedal (promedio $\pm \mathrm{DE}$ ). (C) Diversidad de especies de insectos por humedal (promedio $\pm \mathrm{DE})$. Diferentes superíndices indican diferencias significativas luego de un test de Tukey $(\mathrm{P}<0,05)$.

(A) Insect's species richness for wetlands (mean \pm SD). (B) Insect's abundance for wetlands (mean \pm SD). (C) Insect's species diversity for wetlands (mean \pm SD). Different superscript letters indicate significant differences following a Tukey test $(\mathrm{P}<0.05)$. 

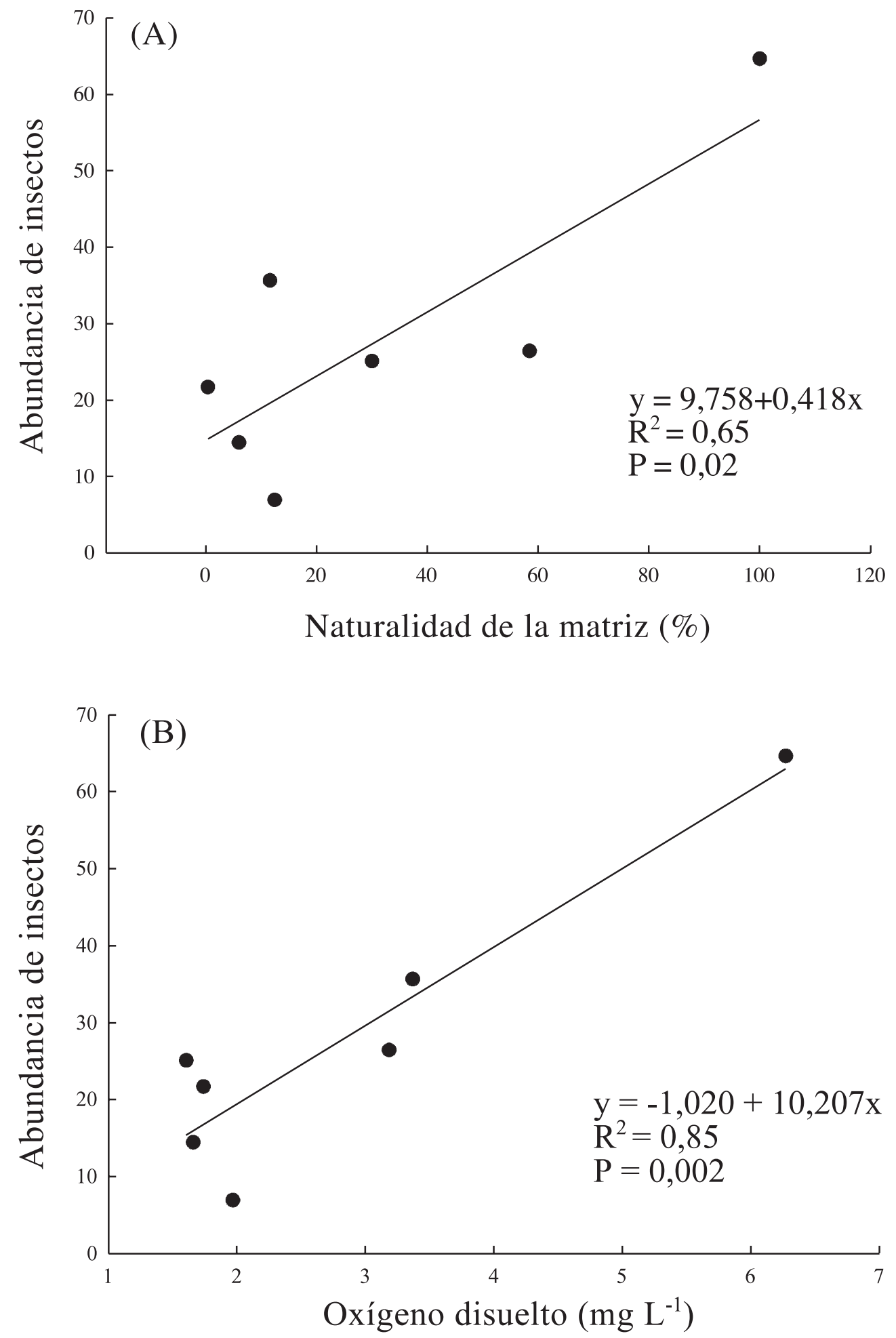

Fig. 4: Relación entre la abundancia de insectos (promedio de las 30 muestras): (A) el porcentaje de naturalidad de la matriz, (B) la concentración de oxígeno en el agua.

Relationship between insect abundance (mean in 30 samples): (A) matrix pristinness, (B) the oxygen concentration in water. 

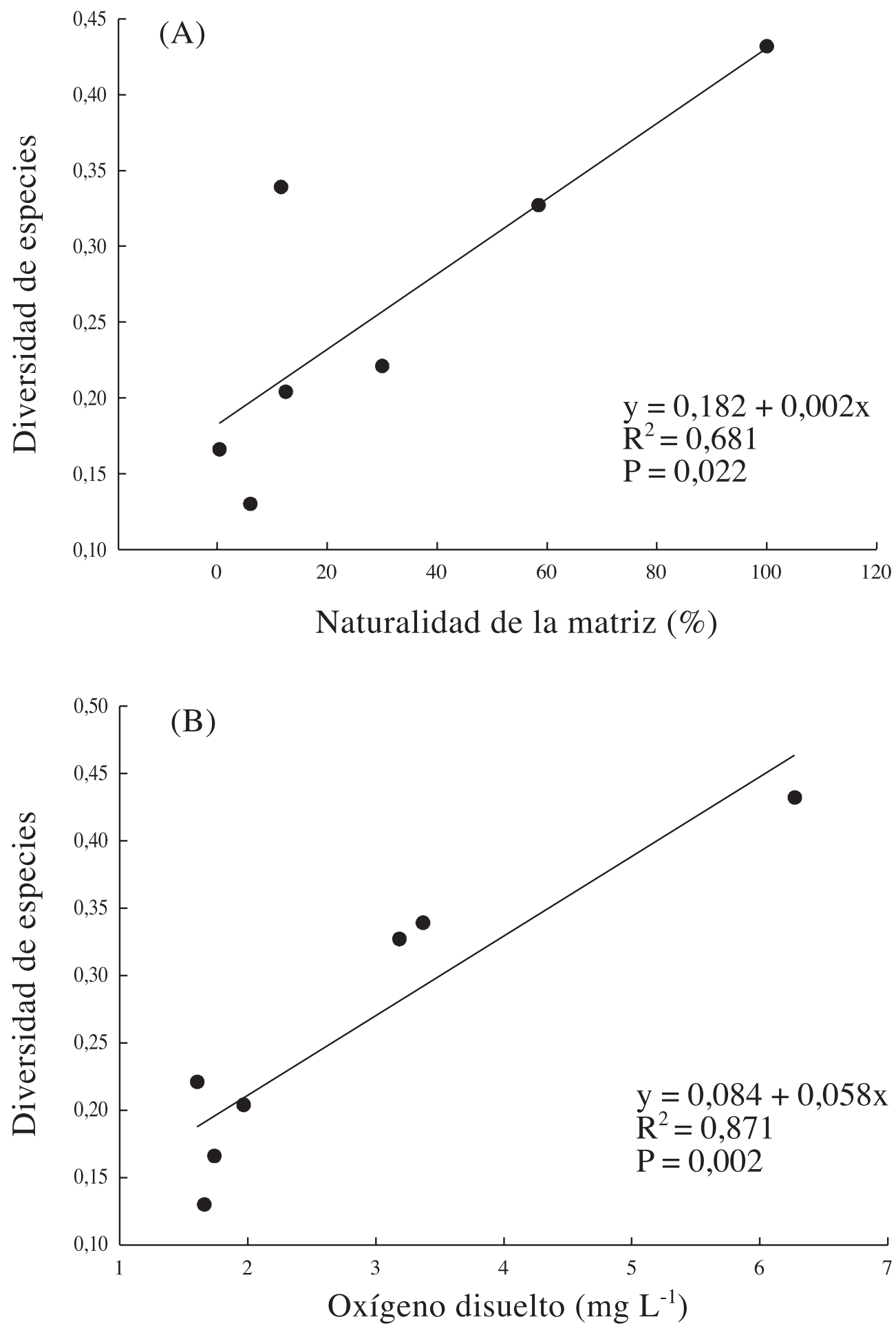

Fig. 5: Relación entre la diversidad de especies de insectos: (A) la naturalidad de la matriz, (B) la concentración de oxígeno en el agua.

Relationship between insect species diversity: (A) matrix pristinness, (B) the oxygen concentration in water. 
TABLA 3

Modelos de regresión múltiple paso a paso entre la estructura del ensamble de insectos (abundancia relativa y diversidad de especies) y la abundancia de especies con las características del hábitat.

NATMAT: naturalidad de la matriz; OXIG: oxígeno disuelto; HETVEG: heterogeneidad vegetacional

Stepwise regression models of the relationship among insect assemblage structure (insect abundance and species diversity) and species abundance with habitat characteristics. NATMAT: matrix naturalness, OXIG: oxygen concentration, HETVEG: vegetation heterogeneity

\begin{tabular}{llcl}
\hline Variable respuesta & Modelo & Valor de $\mathrm{R}^{2}$ & Valor de P \\
\hline Abundancia total & $\mathrm{Y}=-1,026+10,207 \mathrm{OXIG}$ & 0,856 & 0,003 \\
Diversidad de especies & $\mathrm{Y}=0,084+0,058 \mathrm{OXIG}$ & 0,871 & 0,002 \\
Abundancia Belostoma sp. & $\mathrm{Y}=-7,29 \mathrm{E}-02+0,679 \mathrm{HETVEG}$ & 0,885 & 0,013 \\
Abundancia Hydroporini gen. sp. & $\mathrm{Y}=-0,447+0,770 \mathrm{HETVEG}+0,163 \mathrm{OXIG}$ & 0,971 & 0,001 \\
Abundancia Hydrochus sp. & $\mathrm{Y}=-0,356+4,822 \mathrm{E}-02 \mathrm{NATMAT}$ & 0,711 & 0,017 \\
Abundancia Gymnochthebius sp. & $\mathrm{Y}=-1,797+0,869 \mathrm{OXIG}$ & 0,833 & 0,004 \\
Abundancia Chironomiidae gen. sp. & $\mathrm{Y}=-10,387+8,291 \mathrm{OXIG}$ & 0,985 & 0,00001 \\
Abundancia Verger sp. & $\mathrm{Y}=-3,21 \mathrm{E}-02+2,699 \mathrm{E}-03 \mathrm{NATMAT}$ & 0,918 & 0,001 \\
\hline
\end{tabular}

TABLA 4

Modelos de regresión simple para la abundancia de especies de insectos. NATMAT: naturalidad de la matriz; OXIG: oxígeno disuelto; HETVEG: heterogeneidad vegetacional; COND: conductividad; ALCLUZ: profundidad de alcance de la luz

Simple regression models among insects species abundance. NATMAT: matrix naturalness; OXIG: oxygen concentration; HETVEG: vegetation heterogeneity; COND: conductivity; ALCLUZ: light reach deepness

\begin{tabular}{llll}
\hline Variable respuesta & Modelo & Valor de $\mathrm{R}^{2}$ & Valor de P \\
\hline Abundancia Belostoma sp. & $\mathrm{Y}=0,409+1,327 \mathrm{E}-02 \mathrm{AREA}$ & 0,658 & 0,026 \\
& $\mathrm{Y}=4,836 \mathrm{E}-03+1,088 \mathrm{HETVEG}$ & 0,752 & 0,011 \\
Abundancia Ectemnostega sp. & $\mathrm{Y}=380+-3,90 \mathrm{E}-03 \mathrm{COND}$ & 0,578 & 0,047 \\
Abundancia Hydroporini gen. sp. & $\mathrm{Y}=0,185+1,084 \mathrm{E}-02 \mathrm{NATMAT}$ & 0,730 & 0,014 \\
& $\mathrm{Y}=-0,186+1,074 \mathrm{HETVEG}$ & 0,663 & 0,025 \\
& $\mathrm{Y}=-0,110+0.224 \mathrm{OXIG}$ & 0,681 & 0,022 \\
Abundancia Hydrochus sp. & $\mathrm{Y}=-0,217+0,106 \mathrm{ALCLUZ}$ & 0,763 & 0,010 \\
& $\mathrm{Y}=-0,356+4,8822 \mathrm{E}-02 \mathrm{NATMAT}$ & 0,711 & 0,017 \\
Abundancia Gymnochthebius sp. & $\mathrm{Y}=1,638+0,986 \mathrm{OXIG}$ & 0,651 & 0,028 \\
& $\mathrm{Y}=-0,540+3,842 \mathrm{E}-02 \mathrm{NATMAT}$ & 0,743 & 0,012 \\
Abundancia Chironomiidae gen. sp. & $\mathrm{Y}=-1,797+0,869 \mathrm{OXIG}$ & 0,833 & 0,004 \\
Abundancia Verger sp. & $\mathrm{Y}=2,828+0,327 \mathrm{NATMAT}$ & 0,700 & 0,018
\end{tabular}




\section{Área del humedal}

En ecosistemas terrestres, numerosos estudios han mostrado una relación positiva entre la riqueza y abundancia de insectos (Grez 1997, Bolger et al. 2000, Kruess \& Tscharntke 2000, Steffan-Dewenter \& Tscharnntke 2000, Muriel \& Grez 2002) con el área e irregularidad en la forma de los fragmentos de hábitat; sin embargo, en ecosistemas acuáticos existen pocos estudios que den cuenta de esta relación (Lundkvist et al. 2001, Wettstein \& Schmid 1999). En este trabajo solo la abundancia de Belostoma sp. mostró una relación positiva con el área del humedal; no obstante, esta característica no es la más importante para determinar la abundancia de esta especie. Es probable que la disponibilidad de recursos existentes en los humedales, incluido el hábitat, determinen que tanto el tamaño y la forma del humedal no sean significativos para la riqueza de especies y la abundancia de insectos (Wettstein \& Schmid 1999). Sin embargo, dada la relación positiva entre el área y la heterogeneidad de la vegetación, el tamaño del hábitat podría tener una importancia mayor para el ensamble de insectos, si se consideraran humedales de menor superficie (Lord \& Norton 1990, Grez \& González 1995, Poiani et al. 2000).

\section{Heterogeneidad vegetacional}

La relación positiva entre la heterogeneidad vegetacional y diversidad de insectos ha sido ampliamente demostrada (MacGaha 1952, Murdoch et al. 1972, Soska 1975, Southwood et al. 1979, Kouki 1991a, 1991b, Lodge 1991, Newman 1991, Knops et al. 1999). En contraste, solo unos pocos estudios han señalado que al disminuir la heterogeneidad vegetacional la abundancia de algunas especies de insectos decrece (Grez \& González 1995, Cronin et al. 1998). En este trabajo, las abundancias de Belostoma sp. e Hidroporini gen. sp. fueron relacionadas positivamente con la heterogeneidad vegetacional. Esto puede ser explicado en términos de la hipótesis de "diversidad taxonómica" y/o de "heterogeneidad estructural" (Brose 2003). La primera sugiere que si la diversidad de especies en niveles tróficos inferiores controla la diversidad de niveles tróficos superiores (Hunter \& Price 1992, Siemann 1998), cada especie de productor primario adicional podría tener un consumidor especializado (Murdoch et al. 1972), y este, a su vez, un depredador especializado (Hunter \& Price 1992). La segunda hipótesis sugiere que la heterogeneidad estructural de la vegetación puede aumentar el número de especies de cada nivel trófico (Murdoch et al. 1972, Price et al. 1980, Lawton 1983, Heck \& Crowder 1991, Cronin et al. 1998, Dennis et al. 1998, King \& Brazner 1999). De este modo, al disminuir la heterogeneidad vegetacional, la abundancia de los insectos especialistas puede verse fuertemente afectada (Bach 1980, Cronnin et al. 1998). No obstante lo anterior, Belostoma sp. e Hidroporini gen. sp. han sido descritas como especies de hábitos carnívoros y se desconoce si presentan interacciones específicas con sus presas y hospederos.

Oxígeno disuelto, turbidez y conductividad del agua

Se ha demostrado que el oxígeno disuelto constituye el mejor estimador de la calidad del agua y de la capacidad auto-depurativa del ambiente (Parra 1989, Conzonno 1995, Battle \& Golladay 2001, Beavan et al. 2001, Figueroa et al. 2003). En este estudio, la concentración de oxígeno disuelto constituyó uno de los factores más importantes para explicar la variación en la abundancia y diversidad (D') de insectos, y en la abundancia de Hidroporini gen. sp., Hydrochus sp., Gymnochthebius sp., Chironomidae gen. sp. y Verger sp. Esto concuerda con estudios previos, donde el mejor predictor de la estructura del ensamble de invertebrados acuáticos corresponde a la carga de oxígeno disuelto (Spieles \& Mitsch 2000, Nelson et al. 2000). Estos trabajos postulan que la disminución en la concentración de oxígeno disuelto es causada por la eutroficación artificial de los humedales. En relación a esto último, también se produce un aumento de la turbidez del agua por acumulación de materiales arrastrados por aguas lluvia desde la matriz (Parra 1989, Parra et al. 1989). La relación negativa encontrada entre la turbidez del agua de los humedales y la abundancia de Hidroporini gen. sp. aportaría evidencia para la hipótesis propuesta por estos autores. Por otra parte, el efecto negativo de la conductividad del agua sobre la abundancia de Ectemnostega sp., puede indicar que las actividades humanas 
desarrolladas en la matriz circundante a los humedales no solo estarían provocando la eutroficación artificial de estos ecosistemas, sino también la contaminación de sus aguas. Estudios previos sugieren que valores elevados de conductividad se relacionan con la presencia de contaminantes, los cuales pueden provocar cambios en la estructura de los ensambles de insectos cuando su concentración sobrepasa el nivel de tolerancia de algunas especies (Lobretto \& Tell 1995, Tortorelli \& Hernández 1995, Figueroa et al. 2003).

\section{Consideraciones para la conservación}

Nuestros resultados indican que los insectos asociados a la columna de agua pueden ser utilizados como indicadores de los efectos de la urbanización sobre humedales palustres. Las relaciones encontradas muestran que la pérdida y fragmentación del hábitat (área y naturalidad de la matriz), homogeneización del hábitat (heterogeneidad vegetacional), eutroficación (oxígeno disuelto, turbidez del agua) y contaminación de los humedales (conductividad) se traducen en una disminución en la abundancia y diversidad (D') de insectos. De los siete humedales considerados en este estudio, Laguna Verde constituye el único humedal alejado del núcleo urbano. Este presentó los mayores valores de naturalidad de la matriz y concentración de oxígeno en el agua y la más alta abundancia y diversidad (D') de insectos. En relación a lo anterior, la naturalidad de la matriz y la concentración de oxígeno en el agua constituyen las características más importantes para determinar la estructura del ensamble de insectos. La diversidad de especies (D'), contrario a la riqueza de especies, se relacionó significativamente con las características del hábitat, dada las relaciones existentes entre estas y la abundancia de algunas especies de insectos.

Es importante destacar que las especies de insectos, dado sus requerimientos ecofisiológicos, responden diferencialmente a distintas características del hábitat. De esta manera, la abundancia de solo algunas especies fue significativamente relacionada con los atributos del hábitat considerados en este estudio. Algunos efectos de las características del hábitat que nosotros encontramos fueron consistentes con estudios previos (Lundkvist et al. 2001, Figueroa et al. 2003), sin embargo la forma y profundidad de los humedales, el $\mathrm{pH}$, temperatura y nutrientes no mostraron ser importantes en la estructura del ensamble de insectos, y tampoco tuvieron un efecto significativo sobre la abundancia de las especies (Spieles \& Mitsch 2000, Voelz \& McArthur 2000, Figueroa et al. 2003). En este contexto, especies de hemípteros (Belostoma $\mathrm{sp}$. y Ectemnostega sp.), coleópteros (Hidroporini sp., Hydrochus sp., Gymnochthebius sp.), dípteros (Chrironomiidae sp.) y tricópteros (Verger sp.) acuáticos deben ser considerados taxa potencialmente utilizables como indicadores de las condiciones del hábitat en humedales. En Chile, sin embargo, la falta de estudios biológicos y taxonómicos en insectos asociados a estos ecosistemas (Solervicens 1995) dificulta determinar los requerimientos ecológicos de las especies y poner en práctica su uso como indicadores biológicos. No obstante lo anterior, características del hábitat con efectos significativos a un nivel taxonómico más alto (nivel comunitario) podrían ser consideradas para establecer recomendaciones iniciales de planes de manejo. En este estudio, la mayoría de las especies mostraron una mayor relación con la naturalidad de la matriz y la concentración de oxígeno en el agua, al igual que la abundancia y diversidad de insectos. Esta información es de gran utilidad, si consideramos que los humedales palustres constituyen ecosistemas que sostienen una gran biodiversidad, y que los insectos son claves en el mantenimiento de esta.

En este contexto los humedales palustres de la intercomuna Concepción-Talcahuano-San Pedro son áreas importantes para numerosas especies con problemas de conservación (Jiménez 1999), varias de las cuales son consideradas de importancia internacional (Convención Ramsar 1999); sin embargo, la mayor parte de los humedales presentes en esta zona están siendo reemplazados por áreas urbanas (Pauchard et al. 2005). La aplicación de planes de manejo en estos humedales sería de gran utilidad en la prevención de la pérdida de especies y sus hábitats, en uno de los ecosistemas más intervenidos del mundo y donde alto número de especies se pierden en el corto plazo (Collins \& Thomas 1989, Convención Ramsar 1996). 


\section{AGRADECIMIENTOS}

Agradecemos al Sr. Aníbal Venegas por el apoyo prestado en terreno, al Dr. Juan Carlos Ortiz por su gestión para facilitar los muestreos y a los Drs. Jaime Rau, Viviane Jerez, Claudio Ramírez y Pedro Victoriano por sus sugerencias durante la realización de este estudio. Esta investigación ha sido financiada por el proyecto 203.113.062-1.0 y "Proyecto Instrumental Científico 2001" de la Dirección de Investigación de la Universidad de Concepción.

\section{LITERATURA CITADA}

ANDERSON D \& B VONDRACEK (1999) Insects as indicators of land use in three ecoregions of the prairie pothole region. Wetlands 19: 648-664.

BACH CE (1980) Effect of plant density and diversity on the population dynamics of specialist herbivore, the striped cucumberbeetle Acalymma vittata. Ecology 61: $1515-1530$

BARBOSA O \& PA MARQUET (2002) Effects of forest fragmentation on the beetle assemblage at the relict forest of Fray Jorge, Chile. Oecologia 132: 296306.

BATTLE J \& S GOLLADAY (2001) Water quality and macroinvertebrate assemblages in three types of seasonally inundated lime sink wetlands in southwest Georgia. Journal of freshwater ecology 16: 189-207.

BEAVAN L, J SADLER \& C PINDER (2001) The invertebrate fauna of a physically modified urban river. Hydrobiologia 445: 97-108.

BOLGER DT, AV SUÁREZ, KR CROOKS, SA MORRISON \& TJ CASE (2000) Arthropods in urban habitat fragments in southern California: area, age and edge effects. Ecological Applications 10: $1230-1248$.

BROOKS R (2000) Annual and seasonal variation and the effects on hydroperiod on benthic macroinvertebrates of seasonal forest (vernal) ponds in central Massachusetts, USA. Wetlands 20: 707-715.

BROOKS R (2002) Deep- area- volume and hydroperiod relationships of ephemeral (vernal) forest pools in southern New England. Wetlands 22: 247-255.

BROSE U (2003) Bottom- up control of carabid beetle communities in early succession wetlands: mediated by vegetation structure or plant diversity? Oecologia 135: 407-413.

BROWN K (1997) Diversity, disturbance, and sustainable use of Neotropical forest: insects as indicators for conservation monitoring. Journal of Insect Conservation 1: 25-42.

COLLINS N \& J THOMAS (1989) The Conservation of insects and their habitats. Academic Press, London, United Kigdom. 450 pp.

CONZONNO V (1995) Métodos para análisis químicos. En: Lobretto E \& G Tell (eds) Ecosistemas de aguas continentales: 231-249. Tomo I. Editorial Sur, La Plata, Argentina.

CONVENCIÓN RAMSAR (1996) Manual de la
Convención Ramsar: una guía sobre humedales de importancia internacional. Ediciones Oficina de la Convención Ramsar, Gland, Suiza. 325 pp.

CRONIN G, K WISSING \& D LODGE (1998) Comparative feeding selectivity of herbivorous insects on water lilies: aquatics semi- terrestrial insects and submersed vs floating leaves. Freshwater Biology 39: 243-257.

DENNIS P, MR YOUNG, J GORDON (1998) Distribution and abundance of small insects and arachnids in relation to structural heterogeneity of grazed, indigenous grasslands. Ecological Entomology 23: 253-264.

EHRENFELD JG (2004) The expression of multiple functions in urban forested wetlands. Wetlands 1 : 719-733.

FIGUEROA R, C VALDOVINOS, E ARAYA \& O PARRA (2003) Macroinvertebrados bentónicos como indicadores de calidad del agua de ríos del sur de Chile. Revista Chilena de Historia Natural 76: 275-285.

GALLEGO, JB FERNÁNDEZ, MR GARCÍA-MORA \& F GARCÍA-NOVO (1999) Small wetlands lost: a biological conservation hazard in Mediterranean landscapes. Environmental Conservation 26: 190199.

GIBSON C, V BROWN, L LOSITO \& G MCGAVIN (1992) The response of invertebrate assemblies to grazing. Ecography 15: 166-176.

GLEASON R, N EULLIS, D HUBBARD \& W DUFFY (2003) Effects of sediment load on emergence of aquatic invertebrates and plants from wetland soil egg and seed banks. Wetlands 22: 26-34.

GONZÁLEZ A \& P VITORIANO (2005) Aves de los humedales costeros de la zona de Concepción y alrededores. En: Smith-Ramírez C, J Armesto \& C Valdovinos (eds) Historia, biodiversidad y ecología de los bosques costeros de Chile: 485-497. Universitaria Bosque Nativo, Santiago, Chile.

GOTELLI N \& R COLWELL (2001) Quantifying Biodiversity: procedures and pitfalls in the measurement and comparison of species richness. Ecology Letters 4: 379-391.

GREEN DA \& MG BAKER (2003) Urbanization impacts on habitat and bird communities in a Sonoran desert ecosystem. Landscape and urban planning 63: 225239.

GREZ A (1992) Riqueza de especies de insectos herbívoros y tamaño del parche de vegetación huésped: una contrastación experimental. Revista Chilena de Historia Natural 65: 115-120.

GREZ A \& RH GONZÁLEZ (1995) Resource concentration hypothesis: effect of host plant size on density of herbivorous insects. Oecologia 103: 471-474.

GREZ A (1997) Effect of habitat subdivision on the population dynamics of herbivorous and predatory insects in central Chile. Revista Chilena de Historia Natural 70: 481-490.

HECK K \& L CROWDER (1991) Habitat structure and predator- prey interactions in vegetated aquatic systems. En: Bell S, E McCoy \& H Mushinsky (eds) Habitat structure: the physical arrangement of objects in space: 281-299. Chapman \& Hall, London, United Kingdom.

HOLLAND CC, JE HOMEA, SE GWIN \& ME KENTULA (1995) Wetland degradation and loss in the rapidly urbanizing area of Portland, Oregon. Wetlands 15: 336-345.

HUNTER M \& P PRICE (1992) Playing chutes and ladres: 
heterogeneity and the relative roles of bottom-up and top-down forces in natural communities. Ecology 73: 724-732.

HUNTER M (2002) Landscape structure, habitat fragmentation, and the ecology of insects. Agricultural and Forest Entomology 4: 159-166.

JIMÉNEZ M (1999) Evaluación del estado de conservación de las aves de humedal de la Región del Biobío. Unidad de Recursos Renovables, Comisión Nacional del Medio Ambiente, Región del Biobío, Gobierno de Chile, Concepción, Chile. $37 \mathrm{pp}$.

KENTULA ME, EG STEPHANIE \& SM PIERSON (2004) Tracking changes in wetlands with urbanization: sixteen years of experience in Portland, Oregon, USA. Wetlands 1: 734-743.

KING R \& J BRAZNER (1999) Coastal wetland insect communities along a trophic gradient in green bay, Lake Michigan. Wetlands 19:1007-1023.

KITAHARA M \& K SEI (2001) A comparison of the diversity and structure of butterfly communities in semi-natural and human-modified grassland habitat at the foot of Mt. Fuji, central Japan. Biodiversity and Conservation 10: 331-351.

KNOPS J, D TILMAN, N HADDAD, SH NAEEM, CH MITCHELL, J HARSTAD, E RITCHIE, M KNUTSON, J SAUER, D OLSEN, M MOSSMAN, HEMESAATH \& M LANOO (1999) Effects of Landscape Composition and wetland fragmentation on frog and toad abundance and species richness in Iowa and Wisconsin, U.S.A. Conservation Biology 13: $1437-1446$.

KREBS C (1999) Ecological methodology. Benjamin/ Cummnings imprint, California, USA. 620 pp.

KOUKI J (1991a) Small-scale distributional dynamics of the yellowater. Lily and its herbivore Galerucella nymphaceae (Coleoptera: Chrysomelidae). Oecologia 88: 48-54.

KOUKI J (1991b) Tracking spatially variable resources: An experimental study on the oviposition of the water lily beetle. Oikos 61: 243-249.

KRUESS A \& T TSCHARNTKE (2000) Species richness and parasitism in a fragmented landscape: experiments and fields studies with insects on Vicia sepium. Oecologia 122: 129-137.

LAWTON J (1983) Plant architecture and the diversity of phytophagus insects. Annual Review of Entomology 28: 23-39.

LESLIE A, TH CRISMAN, J PRENGER \& K EWEL (1997) Benthic macroinvertebrates of small Florida pondcypress swamps and the influence of dry periods. Wetlands 17: 447-455.

LOBRETTO E \& G TELL (1995) Ecosistemas de aguas continentales. Tomo III. Ediciones Sur, La Plata, Argentina. $1.401 \mathrm{pp}$.

LODGE D (1991) Herbivory of freshwater macrophytes. Aquatic Botany 41: 195-224.

LORD J \& DA NORTON (1990) Scale and the spatial concept of fragmentation. Conservation Biology 28: 287-324.

LUNDKVIST E, J LANDIN \& P MILBERG (2001) Diving beetle (Dytiscidae) assemblages along environmental gradients in an agricultural landscape in southeastern Sweden. Wetlands 21: 48-58.

MAES D, W VANREUSEL, W TALLOEN \& H VAN DICK (2005) Functional conservation units for the endangered Alcon Blue Butterfly Maculinea alcon in Belgium (Lepidoptera: Lycaenidae). Biological Coservation 120: 229-241
MARGALEF (1983) Limnología. Ediciones Omega S.A., Barcelona, España. 1.010 pp

MARZLUFF JM \& K EWING (2001) Restoration of fragmented landscapes for the conservation of birds: A general framework and specific recommendations for urbanizing landscapes. Restoration Ecology 9: 280-292.

MCDONNELL M J \& SA PICKETT (1990) Ecosystem structure and function along urban-rural gradients: an unexploited opportunity for ecology. Ecology 71: $1232-1237$

MCGAHA Y (1952) The limnological relations of insects to certain flowering plants. Transactions of the American Microscopical Society 71: 355-381.

MURDOCH W, F EVANS, CH PETERSON (1972) Diversity and Pattern in plants and insects. Ecology 53: 819-829.

MURIEL SB \& A GREZ (2002) Effect of plant patch shape on the distribution and abundance of three lepidopteran species associated with Brassica oleracea. Agricultural and Forest Entomology 4: 1-7.

NEWMAN R (1991) Herbivory and detritivory on freshwater macrophytes by invertebrates: a review. Journal of the North American Benthological Society 10: 89-114.

NELSON M, A ROLINE, J THULLEN, J SARTORIS \& J BOUTWELL (2000) Invertebrate assemblages and trace element bioaccumulation associated with constructed wetlands. Wetlands 20: 406-415.

PARRA O, V DELLAROSSA, M CONEJEROS, H CAMPOS \& W STEFFENS (1989) Estado de eutrofización de las lagunas Grande de San Pedro y las Tres Pascualas. Universidad de Concepción. Ediciones especiales, Europa-Latinoamérica (EULA), Universidad de Concepción, Concepción, Chile. 174pp.

PARRA O (1989) La eutrofización de la Laguna Grande de San Pedro, Concepción, Chile: un caso de estudio. Ambiente y Desarrollo (Chile) 1: 117-136

PAUCHARD A, M AGUAYO, E PEÑA \& R URRUTIA (in press) Multiple effects of urbanization on the biodiversity of developing countries: the case of a fast-growing metropolitan area (Concepción, Chile). Biological Conservation 127: 272- 281.

POIANI K, B RICHTER, M ANDERSON \& H RICHTER (2000) Biodiversity conservation at multiple scales: functional sites, landscape, and networks. BioScience 50: 133-146.

POLYMERIS C (1995) Vegetación actual de la Península de Hualpén: clasificación y dinámica. Tesis para optar al grado de Magíster en Ciencias con mención en Botánica, Departamento de Botánica, Universidad de Concepción, Concepción, Chile. 190 pp.

PRICE W, C BOUTON, P GROSS, B MCPHERON, J THOMPSOM \& A WIENS (1980) Interaction in the three trophic levels: influence of plants on interactions between herbivores and natural enemies. Annual Review Ecology and Systematics 11. 41-65.

RAU J \& A GANTZ (2001) Fragmentación del bosque nativo del sur de Chile: efectos del área y de la forma sobre la diversidad de aves. Boletín de la Sociedad de Biología de Concepción (Chile) 72: 109-119.

RICCIARDI A, RJ NEVES \& JB RASMUSSEN (1998) Impending extinctions of North American freshwater mussels (Unionoida) following the zebra mussel (Dreissena polymorpha) invasion. Journal of Animal Ecology 67: 613-619.

RIFFO R \& C VILLARROEL (2000) Caracterización de la 
flora y fauna del humedal Los Batros, comuna de San Pedro de la Paz. Gayana (Chile): 64: 23-37.

RIVEROS G, Y SERE \& P DROUILLY (1981) Estructura y diversidad de la comunidad de aves acuáticas de la laguna El Peral, Chile central. Anales del Museo Nacional de Historia Natural 14: 189-196.

RUBBO MJ \& JM KIESECKER (2005) Amphibian breeding distribution in an urbanized landscape. Conservation Biology 19: 504-511.

SEMLITSCH RD \& JR BODIE (1998) Are small, isolated wetlands expendable? Conservation Biology: 11291133

SIEMANN E (1998) Experimental test of effects of plant productivity and diversity on grassland arthropod diversity. Ecology 79: 2057-2070.

SIMONETTI JA (1999) Diversity and conservation of terrestrial vertebrates in Mediterranean Chile. Revista Chilena de Historia Natural 72: 493-500.

SOSKA G (1975) Ecological relations between invertebrates and submerged macrophytes in lake litoral. Ekologia Polska 23: 393-415.

SPIELES D \& W MITSCH (2000) Macroinvertebrate community structure in high and low nutrient constructed wetlands. Wetlands 20: 716-729.

SOLERVICENS J (1995) Consideraciones generales sobre los insectos, el estado de su conocimiento y las colecciones. En: Simonetti J, MTK Arroyo, AE Spotorno \& E Lozada (eds) Diversidad biológica de Chile: 198-210. Comisión Nacional de Ciencia y Tecnología, Santiago, Chile.
SOUTHWOOD T, V BROWN \& P READER (1979) The relationships of plants and insect diversities in succession. Biological Journal of the Linnean Society $12: 327-348$.

SUMMERVILLE K0, L RITTER \& C THOMAS (2004) Forest moth taxa indicators of lepidopteran richness and habitat disturbance: a preliminary assessment. Biological Conservation 116: 9-18.

STEFFAN-DEWENTER I \& T TSCHARNTKE (2000) Butterfly community structure in fragmented habitats. Ecology Letters 3: 449-456.

TAKAMI Y, CH KOSHIO, M ISHII, H FUJII, T HIDAKA \& I SHIMIZU (2004) Genetic diversity and structure of urban populations of Pieris butterflies assessed using amplified fragment length polymorphism. Molecular Ecology 13: 245-258.

TORTORELLI M \& D HERNÁNDEZ (1995) Calidad del agua en un ambiente acuático sometido a efluentes contaminantes. En: Lobretto E \& G Tell (eds) "Ecosistemas de aguas continentales", Tomo I. Editorial Sur, La Plata, Argentina. Pp 227- 230.

VOELZ NJ \& V MCARTHUR (2000) An exploration of factors influencing lotic insect species richness. Biodiversity and Conservation 9: 1543-1570.

WETTSTEIN W \& B SCHMID (1999) Conservation of arthropod diversity in montane wetlands: effect of altitude, habitat quality and habitat fragmentation on butterflies and grass hoppers. Journal of Applied Ecology 36: 365-373. 